Original Research Paper

\title{
Product Moments of Sample Variances and Correlation for Variables with Bivariate Normal Distribution
}

\author{
Juan Romero-Padilla \\ LNPP-Conacyt, Center for Research and Teaching in Economics (CIDE), Mexico City, Mexico
}

Article history

Received: 25-11-2015

Revised: 08-03-2016

Accepted: 10-03-2016

Email: juan.romero@cide.edu

\begin{abstract}
A general result to obtain the product moments of two sample variances and the sample correlation when the data follow a bivariate normal distribution is derived; the result is expressed in terms of the hypergeometric function. As corollaries, two general equations are stated, one to obtain the moments of the correlation sample and one to obtain the moments of the ratio of two sample variances. To evaluate the product moments in short closed forms, three theorems have been established. The results are used to obtain the expectation and variance for the ratio of two correlated sample variances. Finally, some examples of particular product moments are provided and some validations were carried out.
\end{abstract}

Keywords: Wishart Distribution, Product Moments, Hypergeometric Function, Sample Correlation Coefficient, Sample Variance

\section{Introduction}

We are interested in the product moments of the two sample variances $\left(S_{1}^{2}, S_{2}^{2}\right)$ and sample correlation coefficient $(R)$ of the bivariate normal distribution, so we want to derive $E\left(S_{1}^{2 a} S_{2}^{2 b} R^{c}\right)$ for finite $a, b, c$. One approach to obtain the product moments of the two sample variances and the correlation coefficient was discussed by Joarder (2006), however his results involves an infinite series that does not consider an important term, without the missing term, the result of Joarder (2006) does not work to get some product moments, for instance, we are not able to get the first moment of R. Here we expand the result of Joarder (2006) to derive a more general result.

There are different expressions for moments of $\mathrm{R}$, Ghosh (1966) and Rady et al. (2005) obtained the first four moments of $\mathrm{R}$, here we derived a general equation for any moment of $\mathrm{R}$ that do not involves an infinite sum and it is expressed in terms of the well-known hypergeometric function.

Let $X_{1}, \ldots, X_{n}$ be iid $N_{p}(\mu, \Sigma)$ where $\Sigma$ is positive defined and $n>p$, the sums of squares and cross product matrix is given by:

$$
A=\sum_{j=1}^{n}\left(X_{j}-\bar{X}\right)\left(X_{j}-\bar{X}\right)^{T}
$$

$A$, is said to have a Wishart distribution with parameters $p, m=n-1$ and $\Sigma(p x p), A \sim W_{p}(m, \Sigma)$ and the
Probability Density Function (PDF) is given by (Anderson, 2003):

$$
f(A)=\frac{|A|^{\frac{(m-p-1)}{2}} \exp \left(-\frac{1}{2} \operatorname{tr} \Sigma^{-1} A\right)}{2^{\frac{m p}{2}} \pi^{\frac{p(p-1)}{2}}|\Sigma|^{\frac{m}{2}} \prod_{i=1}^{p} \Gamma\left(\frac{1}{2}(m+1-i)\right)}
$$

With, $m>p$ and $A$ positive definite.

For $p=2$ we have the bivariate case with:

$$
A=\left[\begin{array}{ll}
a_{11} & a_{12} \\
a_{12} & a_{22}
\end{array}\right]
$$

Where:

$$
\begin{aligned}
& a_{11}=m S_{1}^{2}, a_{22}=m S_{2}^{2}, a_{12}=m S_{12}=m r S_{1} S_{2}, \\
& R=\frac{S_{12}}{S_{1} S_{2}}=\frac{a_{12}}{\left(a_{1} a_{2}\right)^{1 / 2}}
\end{aligned}
$$

$S_{12}$ is the sample covariance and the joint pdf of $a_{11}$, $a_{22}$ and $a_{12}$ is given by:

$$
\begin{aligned}
& f\left(a_{11}, a_{22}, a_{12}\right)=\frac{\left(1-\rho^{2}\right)^{-\frac{m}{2}}\left(\sigma_{1} \sigma_{2}\right)^{-m}}{2^{m} \pi^{1 / 2} \Gamma\left(\frac{m}{2}\right) \Gamma\left(\frac{m-1}{2}\right)}\left(a_{11} a_{22}-a_{12}^{2}\right)^{\frac{m-3}{2}} \\
& \times \exp \left(-\frac{1}{2}\left[\frac{a_{11}}{\left(1-\rho^{2}\right) \sigma_{1}^{2}}+\frac{a_{22}}{\left(1-\rho^{2}\right) \sigma_{2}^{2}}+\frac{2 \rho a_{12}}{\left(1-\rho^{2}\right) \sigma_{1} \sigma_{2}}\right]\right)
\end{aligned}
$$




\section{Product Moments of Sample Variances and Sample Correlation}

Throughout the paper, we will use the generalized hypergeometric function (e.g., Bailey, 1964):

$$
{ }_{p} F_{q}\left(a_{1}, \ldots, a_{p}: b_{1}, \ldots, b_{q} ; z\right)=\sum_{k=0}^{\infty} \frac{\left(a_{1}\right)_{k}\left(a_{2}\right)_{k} \ldots\left(a_{p}\right)_{k} z^{k}}{\left(b_{1}\right)_{k}\left(b_{2}\right)_{k} \ldots\left(b_{q}\right)_{k} k !}
$$

With:

$$
(a)_{0}=1 ;(a)_{k}=(a+k-1)(a+k-2) \ldots(a+1) a=\frac{\Gamma(a+k)}{\Gamma(a)}
$$

Some properties of the hypergeometric function (Oberhettinger, 1970):

$$
\begin{aligned}
& { }_{2} F_{1}(a, b ; b ; z)={ }_{1} F_{0}(a ; z)=(1-z)^{-a}, \\
& { }_{2} F_{1}(a, b ; c ; z)=(1-z)^{c-a-b}{ }_{2} F_{1}(c-a, c-b ; c ; z), \\
& \frac{d}{d z}{ }_{2} F_{1}(a, b ; c ; z)=\frac{a b}{c}{ }_{2} F_{1}(a+1, b+1 ; c+1 ; z)
\end{aligned}
$$

And the following results:

$$
\begin{aligned}
& \int_{0}^{\pi / 2} \sin ^{x} \theta d \theta=\frac{\sqrt{\pi} \Gamma\left(\frac{x+1}{2}\right)}{2 \Gamma\left(\frac{x}{2}+1\right)}, \\
& \int_{0}^{\infty} t^{x} e^{-y t} d t=\frac{\Gamma(x+1)}{y^{x+1}}, \\
& \Gamma\left(\frac{1}{2}+k\right)=\frac{\sqrt{\pi}(2 k-1) !}{2^{2 k-1}(k-1) !}=\frac{\sqrt{\pi}(2 k) !}{2^{2 k} k !}
\end{aligned}
$$

where, $\Gamma($.$) is the gamma function.$

\section{Theorem 1}

For any finite $a, b$ and $c$. If $c$ is even, the product moments of the sample variances and sample correlation, $E\left(S_{1}^{2 a} S_{2}^{2 b} R^{c}\right)$, are given by:

$$
\begin{aligned}
& E\left[S_{1}^{2 a} S_{2}^{2 b} R^{c}\right]=\frac{2^{a+b}\left(1-\rho^{2}\right)^{a+b+\frac{m}{2}}}{\sqrt{\pi} \Gamma\left(\frac{m}{2}\right) m^{a+b}} \sigma_{1}^{2 a} \sigma_{2}^{2 b} \\
& \times \Gamma\left(\frac{m+2 a}{2}\right) \Gamma\left(\frac{m+2 b}{2}\right) \Gamma\left(\frac{c+1}{2}\right) \Gamma^{-1}\left(\frac{m+c}{2}\right) \\
& \times{ }_{3} F_{2}\left(\frac{m+2 a}{2}, \frac{m+2 b}{2}, \frac{c+1}{2} ; \frac{m+c}{2}, \frac{1}{2} ; \rho^{2}\right)
\end{aligned}
$$

If $\mathrm{c}$ is odd are given by:

$$
\begin{aligned}
& E\left[S_{1}^{2 a} S_{2}^{2 b} R^{c}\right]=\frac{2^{a+b-1}\left(1-\rho^{2}\right)^{a+b+\frac{m}{2}}}{\sqrt{\pi} \Gamma\left(\frac{m}{2}\right) m^{a+b}} \sigma_{1}^{2 a} \sigma_{2}^{2 b} \rho \\
& \times \Gamma\left(\frac{m+1+2 a}{2}\right) \Gamma\left(\frac{m+1+2 b}{2}\right) \Gamma\left(\frac{c+2}{2}\right) \Gamma^{-1}\left(\frac{m+1+c}{2}\right) \\
& \times{ }_{3} F_{2}\left(\frac{m+1+2 a}{2}, \frac{m+1+2 b}{2}, \frac{c+2}{2} ; \frac{m+1+c}{2}, \frac{3}{2} ; \rho^{2}\right)
\end{aligned}
$$

\section{Proof}

We use the joint distribution of $S_{1}^{2}, S_{2}^{2}$ and $R$ given by Joarder (2006):

$$
\begin{aligned}
& f\left(S_{1}^{2}, S_{2}^{2}, R\right)=\frac{m^{m}\left(1-\rho^{2}\right)^{-\frac{m}{2}}\left(\sigma_{1} \sigma_{2}\right)^{-m}}{4 \pi \Gamma(m-1)} \\
& \times S_{1}^{m-2} S_{2}^{m-2}\left(1-R^{2}\right)^{\frac{m-3}{2}} \\
& \times \exp \left(-\frac{1}{2}\left[\frac{m S_{1}^{2}}{\left(1-\rho^{2}\right) \sigma_{1}^{2}}+\frac{m S_{2}^{2}}{\left(1-\rho^{2}\right) \sigma_{2}^{2}}+\frac{2 \rho m R S_{1} S_{2}}{\left(1-\rho^{2}\right) \sigma_{1} \sigma_{2}}\right]\right)
\end{aligned}
$$

The product moments, for any $a, b, c$ are given by:

$$
\begin{aligned}
& E\left(S_{1}^{2 a} S_{2}^{2 b} R^{c}\right)=\int_{-1}^{1} \int_{0}^{\infty} \int_{0}^{\infty} S_{1}^{2 a} S_{2}^{2 b} R^{c} f\left(S_{1}^{2}, S_{2}^{2}, R\right) d S_{1}^{2} d S_{2}^{2} d R \\
& =\frac{m^{m}\left(1-\rho^{2}\right)^{-\frac{m}{2}}\left(\sigma_{1} \sigma_{2}\right)^{-m}}{4 \pi \Gamma(m-1)} \sum_{k=0}^{\infty} \frac{(m \rho)^{k}}{k !\left(1-\rho^{2}\right)^{k}\left(\sigma_{1} \sigma_{2}\right)^{k}} \\
& \times \int_{0}^{\infty} S_{1}^{m+k+2 a-2} \exp \left(-\frac{m S_{1}^{2}}{2\left(1-\rho^{2}\right) \sigma_{1}^{2}}\right) d S_{1}^{2} \\
& \times \int_{0}^{\infty} S_{2}^{m+k+2 b-2} \exp \left(-\frac{m S_{2}^{2}}{2\left(1-\rho^{2}\right) \sigma_{2}^{2}}\right) d S_{2}^{2} \\
& \times\left[\int_{-1}^{1} R^{k+c}\left(1-R^{2}\right)^{(m-3) / 2} d R\right. \\
& \left.+\int_{0}^{1} R^{k+c}\left(1-R^{2}\right)^{(m-3) / 2} d R\right]
\end{aligned}
$$

The first two integrals of Equation 6 can be expressed as:

$$
\begin{aligned}
& \int_{0}^{\infty} S_{1}^{m+k+2 a-2} \exp \left(-\frac{m S_{1}^{2}}{2\left(1-\rho^{2}\right) \sigma_{1}^{2}}\right) d S_{1}^{2} \\
& \times \int_{0}^{\infty} S_{2}^{m+k+2 b-2} \exp \left(-\frac{m S_{2}^{2}}{2\left(1-\rho^{2}\right) \sigma_{2}^{2}}\right) d S_{2}^{2} \\
& =\Gamma\left(\frac{k+m}{2}+a\right) \Gamma\left(\frac{k+m}{2}+b\right)
\end{aligned}
$$




$$
\begin{aligned}
& \times\left[\left(\frac{m}{2\left(1-\rho^{2}\right) \sigma_{1}^{2}}\right)^{\frac{m+k}{2}+a}\left(\frac{m}{2\left(1-\rho^{2}\right) \sigma_{2}^{2}}\right)^{\frac{m+k}{2}+b}\right]^{-1} \\
& =\Gamma\left(\frac{k+m}{2}+a\right) \Gamma\left(\frac{k+m}{2}+b\right) \\
& \times 2^{m+k+a+b}\left(1-\rho^{2}\right)^{m+k+a+b} \sigma_{1}^{m+k+2 a} \sigma_{2}^{m+k+2 b} \\
& \times\left(m^{m+k+a+b}\right)^{-1}
\end{aligned}
$$

To obtain the last two integrals of Equation 6 we use the beta function:

$$
\begin{aligned}
& {\left[\int_{-1}^{0} R^{k+c}\left(1-R^{2}\right)^{\frac{m-3}{2}} d R+\int_{0}^{1} R^{k+c}\left(1-R^{2}\right)^{\frac{m-3}{2}} d R\right]} \\
& =\left[(-1)^{k+c}+1\right] \int_{0}^{1} R^{k+c}\left(1-r^{2}\right)^{\frac{m-3}{2}} d R \\
& =\left[(-1)^{k+c}+1\right] \frac{1}{2} \int_{0}^{1} w^{\frac{k+c-1}{2}}(1-w)^{\frac{m-3}{2}} d w \\
& =\frac{1}{2}\left[(-1)^{k+c}+1\right] B\left(\frac{k+c+1}{2}, \frac{m-1}{2}\right)
\end{aligned}
$$

The product moments are given by the expression:

$$
\begin{aligned}
& E\left[S_{1}^{2 a} S_{2}^{2 b} R^{c}\right]=\frac{2^{a+b}\left(1-\rho^{2}\right)^{a+b+\frac{m}{2}}}{\sqrt{\pi} \Gamma\left(\frac{m}{2}\right) m^{a+b}} \sigma_{1}^{2 a} \sigma_{2}^{2 b} \\
& \times \sum_{k=0}^{\infty} \frac{1}{2}\left[(-1)^{k+c}+1\right] \frac{(2 \rho)^{k}}{k !} \Gamma\left(\frac{k+m}{2}+a\right) \\
& \times \Gamma\left(\frac{k+m}{2}+b\right) \Gamma\left(\frac{k+c+1}{2}\right) \Gamma^{-1}\left(\frac{k+m+c}{2}\right)
\end{aligned}
$$

To express $E\left[S_{1}^{2 a} S_{2}^{2 b} R^{c}\right]$ in terms of hypergeometric function we have two cases, if $c$ is even redefine $k=2 j$ then $\frac{1}{2}\left[(-1)^{2 j+c}+1\right]=1$ and:

$$
\begin{aligned}
& E\left[S_{1}^{2 a} S_{2}^{2 b} R^{c}\right]=\frac{2^{a+b}\left(1-\rho^{2}\right)^{a+b+\frac{m}{2}}}{\sqrt{\pi} \Gamma\left(\frac{n}{2}\right) m^{a+b}} \sigma_{1}^{2 a} \sigma_{2}^{2 b} \\
& \times \sum_{j=0}^{\infty} \frac{\sqrt{\pi}(\rho)^{2 j}}{j !} \Gamma\left(\frac{m}{2}+j+a\right) \Gamma\left(\frac{m}{2}+j+b\right) \\
& \times \Gamma\left(\frac{c+1}{2}+j\right) \Gamma^{-1}\left(\frac{m+c}{2}+j\right) \Gamma^{-1}\left(\frac{1}{2}+j\right) \\
& =\frac{2^{a+b}\left(1-\rho^{2}\right)^{a+b+\frac{m}{2}}}{\sqrt{\pi} \Gamma\left(\frac{m}{2}\right) m^{a+b}} \sigma_{1}^{2 a} \sigma_{2}^{2 b} \Gamma\left(\frac{m}{2}+a\right)
\end{aligned}
$$

$$
\begin{aligned}
& \times \Gamma\left(\frac{m}{2}+b\right) \Gamma\left(\frac{c+1}{2}\right) \Gamma^{-1}\left(\frac{m+c}{2}\right) \\
& \times \sum_{k=0}^{\infty} \frac{\left(\rho^{2}\right)^{j}}{j !}\left(\frac{m}{2}+a\right)_{j}\left(\frac{m}{2}+b\right)_{j}\left(\frac{c+1}{2}\right)_{j}\left[\left(\frac{m+c}{2}\right)_{j}\left(\frac{1}{2}\right)_{j}\right]^{-1} \\
& =\frac{2^{a+b}\left(1-\rho^{2}\right)^{a+b+\frac{m}{2}}}{\sqrt{\pi} \Gamma\left(\frac{m}{2}\right) m^{a+b}} t \sigma_{1}^{2 a} \sigma_{2}^{2 b} \Gamma\left(\frac{m}{2}+a\right) \\
& \times \Gamma\left(\frac{m}{2}+b\right) \Gamma\left(\frac{c+1}{2}\right) \Gamma^{-1}\left(\frac{m+c}{2}\right) \\
& \times{ }_{3} F_{2}\left(\frac{m+2 a}{2}, \frac{m+2 b}{2}, \frac{c+1}{2} ; \frac{m+c}{2}, \frac{1}{2} ; \rho^{2}\right)
\end{aligned}
$$

If $\mathrm{c}$ is odd redefine $k=2 j-1$ then $\frac{1}{2}\left[(-1)^{2 j-1+c}+1\right]=1$ and:

$$
\begin{aligned}
& E\left[S_{1}^{2 a} S_{2}^{2 b} R^{c}\right]=\frac{2^{a+b}\left(1-\rho^{2}\right)^{a+b+\frac{m}{2}}}{\sqrt{\pi} \Gamma\left(\frac{m}{2}\right) m^{a+b}} \sigma_{1}^{2 a} \sigma_{2}^{2 b} \\
& \times \sum_{j=1}^{\infty} \frac{(2 \rho)^{2 j-1}}{(2 j-1) !} \Gamma\left(\frac{m-1}{2}+j+a\right) \Gamma\left(\frac{m-1}{2}+j+b\right) \\
& \times \Gamma\left(\frac{c}{2}+j\right) \Gamma^{-1}\left(\frac{m-1+c}{2}+j\right) \Gamma^{-1}\left(\frac{1}{2}+j\right) \frac{\sqrt{\pi}(2 j-1) !}{2^{2 j-1}(j-1) !} \\
& =\frac{2^{a+b}\left(1-\rho^{2}\right)^{a+b+\frac{m}{2}}}{\Gamma\left(\frac{n}{2}\right) m^{a+b}} \sigma_{1}^{2 a} \sigma_{2}^{2 b} \rho \\
& \times \sum_{j=1}^{\infty} \frac{\left(\rho^{2}\right)^{j-1}}{(j-1) !} \Gamma\left(j-1+\frac{m+1}{2}+a\right) \Gamma\left(j-1+\frac{m+1}{2}+b\right) \\
& \times \Gamma\left(j-1+\frac{c+2}{2}\right) \Gamma^{-1}\left(j-1+\frac{m+c+1}{2}\right) \Gamma^{-1}\left(j-1+\frac{3}{2}\right)
\end{aligned}
$$

Let $h=j-1$, then:

$$
\begin{aligned}
& E\left[S_{1}^{2 a} S_{2}^{2 b} R^{c}\right]=\frac{2^{a+b}\left(1-\rho^{2}\right)^{a+b+\frac{m}{2}}}{\Gamma\left(\frac{m}{2}\right) m^{a+b}} \sigma_{1}^{2 a} \sigma_{2}^{2 b} \rho \\
& \times \Gamma\left(\frac{m+1}{2}+a\right) \Gamma\left(\frac{m+1}{2}+b\right) \Gamma\left(\frac{c+2}{2}\right) \\
& \times \Gamma^{-1}\left(\frac{m+c+1}{2}\right) \Gamma^{-1}\left(\frac{3}{2}\right) \\
& \times \sum_{h=0}^{\infty} \frac{\left(\rho^{2}\right)^{h}}{h !}\left(\frac{m+1}{2}+a\right)_{h}\left(\frac{m+1}{2}+b\right)_{h} \\
& \times\left(\frac{c+2}{2}\right)_{h}\left[\left(\frac{m+1+c}{2}\right)_{h}\left(\frac{3}{2}\right)_{h}\right]^{-1}
\end{aligned}
$$




$$
\begin{aligned}
& =\frac{2^{a+b+1}\left(1-\rho^{2}\right)^{a+b+\frac{m}{2}}}{\sqrt{\pi} \Gamma\left(\frac{m}{2}\right) m^{a+b}} \sigma_{1}^{2 a} \sigma_{2}^{2 b} \rho \\
& \times \Gamma\left(\frac{m+1}{2}+a\right) \Gamma\left(\frac{m+1}{2}+b\right) \Gamma\left(\frac{c+2}{2}\right) \Gamma^{-1}\left(\frac{m+c+1}{2}\right) \\
& \times{ }_{3} F_{2}\left(\frac{m+1+2 a}{2}, \frac{m+1+2 b}{2}, \frac{c+2}{2} ; \frac{m+1+c}{2}, \frac{3}{2} ; \rho^{2}\right)
\end{aligned}
$$

The following expression will be used in the next two theorems, let:

$$
b_{k, m}=\frac{1}{2}\left[(-1)^{k+c}+1\right] \frac{(2)^{k}}{k !} \Gamma\left(\frac{k+1}{2}\right) \Gamma\left(\frac{k+m}{2}\right), m>2, k>0
$$

\section{Theorem 2}

For $m>2$ and $-1<\rho<1$, we have:

$$
\left(1-\rho^{2}\right)^{-\frac{m}{2}}={ }_{1} F_{0}\left(\frac{m}{2} ; ; \rho^{2}\right)
$$

If $\mathrm{c}$ is even, we have:

$$
\begin{gathered}
\sum_{k=0}^{\infty} \rho^{k} b_{k, m}=\sqrt{\pi} \Gamma\left(\frac{m}{2}\right)\left(1-\rho^{2}\right)^{-\frac{m}{2}} \\
\sum_{k=0}^{\infty} \rho^{k} b_{k, m}\left(\frac{k+m}{2}\right)=\sqrt{\pi} \Gamma\left(\frac{m+2}{2}\right)\left(1-\rho^{2}\right)^{-\frac{m+2}{2}}
\end{gathered}
$$

If $\mathrm{c}$ is odd, we have:

$$
\begin{gathered}
\sum_{k=0}^{\infty} \rho^{k} b_{k, m}=2 \rho \Gamma\left(\frac{m+1}{2}\right)_{2} F_{1}\left(1, \frac{m+1}{2} ; \frac{3}{2} ; \rho^{2}\right) \\
\sum_{k=0}^{\infty} \rho^{k} b_{k, m}\left(\frac{k+m}{2}\right)=\rho \sqrt{\pi} \Gamma\left(\frac{m+1}{2}\right) \Gamma\left(\frac{3}{2}\right) \\
\times\left[{ }_{2} F_{1}\left(2, \frac{m+1}{2} ; \frac{3}{2} ; \rho^{2}\right)-\left(\frac{m-1}{2}\right)_{2} F_{1}\left(1, \frac{m+1}{2} ; \frac{3}{2} ; \rho^{2}\right)\right]
\end{gathered}
$$

\section{Proof}

Note that:

$$
1=E\left[S_{1}^{2(0)} S_{2}^{2(0)} r^{0}\right]=\left(1-\rho^{2}\right)^{\frac{m}{2}}{ }_{1} F_{0}\left(\frac{m}{2} ; ; \rho^{2}\right)
$$

Then:

$$
\left(1-\rho^{2}\right)^{-\frac{m}{2}}=F_{0}\left(\frac{m}{2} ; ; \rho^{2}\right)
$$

If $\mathrm{c}$ is even redefine $k=2 j$ :

$$
\begin{aligned}
\sum_{k=0}^{\infty} \rho^{k} b_{k, m}=\sum_{k=0}^{\infty} \frac{1}{2}\left[(-1)^{k+c}+1\right] \frac{(2 \rho)^{k}}{k !} \Gamma\left(\frac{k+1}{2}\right) \Gamma\left(\frac{k+m}{2}\right) \\
=\sum_{j=0}^{\infty} \frac{(2 \rho)^{2 j}}{(2 j) !} \Gamma\left(j+\frac{1}{2}\right) \Gamma\left(j+\frac{m}{2}\right) \\
=\sqrt{\pi} \sum_{j=0}^{\infty} \frac{(\rho)^{2 j}}{(j) !} \Gamma\left(j+\frac{m}{2}\right) \\
=\sqrt{\pi} \Gamma\left(\frac{m}{2}\right) \sum_{k=0}^{\infty} \frac{\left(\rho^{2}\right)^{j}}{(j) !}\left(\frac{m}{2}\right)_{j} \\
=\sqrt{\pi} \Gamma\left(\frac{m}{2}\right){ }_{1} F_{0}\left(\frac{m}{2} ; ; \rho^{2}\right) \\
\quad \sum_{k=0}^{\infty} \rho^{k} b_{k, m}\left(\frac{k+m}{2}\right)=\sum_{k=0}^{\infty}\left(\frac{k+m}{2}\right) \frac{1}{2}\left[(-1)^{k+c}+1\right] \\
\quad \times \frac{(2 \rho)^{k}}{k !} \Gamma\left(\frac{k+m}{2}\right) \Gamma\left(\frac{k+1}{2}\right) \\
=\left(\frac{m}{2}\right) \sqrt{\pi} \Gamma\left(\frac{m}{2}\right)\left(1-\rho^{2}\right)^{-\frac{m}{2}} \\
\quad+\left(\frac{1}{2}\right) m \rho^{2} \sqrt{\pi} \Gamma\left(\frac{m}{2}\right)\left(1-\rho^{2}\right)^{-\frac{m}{2}-1} \\
\quad=\left(\frac{m}{2}\right) \sqrt{\pi} \Gamma\left(\frac{m}{2}\right)\left(1-\rho^{2}\right)^{-\frac{m}{2}}\left\{1+\rho^{2}\left(1-\rho^{2}\right)^{-1}\right\} \\
\quad=\left(\frac{m}{2}\right) \sqrt{\pi} \Gamma\left(\frac{m}{2}\right)\left(1-\rho^{2}\right)^{-\frac{m+2}{2}}
\end{aligned}
$$

If $c$ is odd redefine $k=2 j-1$ :

$$
\begin{aligned}
& \sum_{k=0}^{\infty} \rho^{k} b_{k, m}=\sum_{j=1}^{\infty} \frac{(2 \rho)^{2 j-1}}{(2 j-1) !} \Gamma(j) \Gamma\left(\frac{2 j-1+m}{2}\right) \\
& \times \Gamma^{-1}\left(\frac{1}{2}+j\right) \frac{\sqrt{\pi}(2 j-1) !}{2^{2 j-1}(j-1) !} \\
& =\sum_{j=1}^{\infty} \frac{\rho \sqrt{\pi}\left(\rho^{2}\right)^{j-1}}{(j-1) !} \Gamma(j-1+1) \\
& \times \Gamma\left(j-1+\frac{m+1}{2}\right) \Gamma^{-1}\left(j-1+\frac{3}{2}\right) \\
& =\rho \sqrt{\pi} \Gamma\left(\frac{m+1}{2}\right) \Gamma^{-1}\left(\frac{3}{2}\right) \\
& \times \sum_{h=0}^{\infty} \frac{\left(\rho^{2}\right)^{h}}{h !}(1)_{h}\left(\frac{m+1}{2}\right)_{h}\left[\left(\frac{3}{2}\right)_{h}\right]^{-1} \\
& =2 \rho \Gamma\left(\frac{m+1}{2}\right)_{2} F_{1}\left(1, \frac{m+1}{2} ; \frac{3}{2} ; \rho^{2}\right)
\end{aligned}
$$

$$
\begin{aligned}
& \sum_{k=0}^{\infty} \rho^{k} b_{k, m}\left(\frac{k+m}{2}\right)=\sum_{j=1}^{\infty}\left(\frac{2 j-1+m}{2}\right) \frac{(2 \rho)^{2 j-1}}{(2 j-1) !} \Gamma(j) \\
& \times \Gamma\left(\frac{2 j-1+m}{2}\right) \Gamma^{-1}\left(\frac{1}{2}+j\right) \frac{\sqrt{\pi}(2 j-1) !}{2^{2 j-1}(j-1) !}
\end{aligned}
$$




$$
\begin{aligned}
& =\sum_{j=1}^{\infty} \rho \sqrt{\pi}\left(\rho^{2}\right)^{j-1} \Gamma(j+1) \\
& \times \Gamma\left(j-1+\frac{m+1}{2}\right) \Gamma^{-1}\left(j-1+\frac{3}{2}\right) \frac{1}{(j-1) !} \\
& -\sum_{j=1}^{\infty}\left(\frac{m-1}{2}\right) \rho \sqrt{\pi}\left(\rho^{2}\right)^{j-1} \Gamma(j-1+1) \\
& \times \Gamma\left(j-1+\frac{m+1}{2}\right) \Gamma^{-1}\left(j-1+\frac{3}{2}\right) \frac{1}{(j-1) !} \\
& =\rho \sqrt{\pi} \Gamma\left(\frac{m+1}{2}\right) \Gamma\left(\frac{3}{2}\right) \\
& \times\left[{ }_{2} F_{1}\left(2, \frac{m+1}{2} ; \frac{3}{2} ; \rho^{2}\right)-\left(\frac{m-1}{2}\right){ }_{2} F_{1}\left(1, \frac{m+1}{2} ; \frac{3}{2} ; \rho^{2}\right)\right]
\end{aligned}
$$

To evaluate the product moments $E\left[S_{1}^{2 a} S_{2}^{2 b} R^{c}\right]$ of some special cases, Joarder (2006) derived a result expressed in his paper by Theorem 3.1, which only holds when $c$ is even and the expression for $b_{k, m}$ is different (Equation 8). The next Theorem 3, considers the case when $c$ is not even.

\section{Theorem 3}

For $m>2$ and $-1<\rho<1$ and $b_{k, m}$ defined in (8), we have for $c$ even:

(i) $\quad \sum_{k=0}^{\infty} k \rho^{k} b_{k, n}=n \rho^{2} \sqrt{\pi} \Gamma\left(\frac{n}{2}\right)\left(1-\rho^{2}\right)^{-\frac{n}{2}-1}$

(ii) $\quad \sum_{k=0}^{\infty} k^{2} \rho^{k} b_{k, m}=\left(m^{2} \rho^{4}+2 m \rho^{2}\right)\left(1-\rho^{2}\right)^{-2} L(m, \rho)$

(iii) $\quad \sum_{k=0}^{\infty} k^{3} \rho^{k} b_{k, m}=w_{3}(m, \rho) L(m, \rho)$

(iv) $\quad \sum_{k=0}^{\infty} k^{4} \rho^{k} b_{k, m}=w_{4}(m, \rho) L(m, \rho)$

$$
\begin{aligned}
& \sum_{k=0}^{\infty} k^{\{2\}} \rho^{k} b_{k, m}=\left(m(m+1) \rho^{4}+m \rho^{2}\right) \\
& \times\left(1-\rho^{2}\right)^{-2} L(m, \rho)
\end{aligned}
$$

(vi) $\quad \sum_{k=0}^{\infty} k^{\{3\}} \rho^{k} b_{k, m}=w_{\{3\}}(m, \rho) L(m, \rho)$

(vii) $\quad \sum_{k=0}^{\infty} k^{\{4\}} \rho^{k} b_{k, m}=w_{\{4\}}(m, \rho) L(m, \rho)$

For $c$ odd, we have:

(viii) $\quad \sum_{k=0}^{\infty} k \rho^{k} b_{k, m}=4 \Gamma\left(\frac{m+1}{2}\right)\left[\frac{\rho}{2} A_{1}+\rho^{3}\left(\frac{m+1}{3}\right) A_{2}\right]$ (ix)

$\sum_{k=0}^{\infty} k^{2} \rho^{k} b_{k, m}=16 \Gamma\left(\frac{m+1}{2}\right)\left[\frac{\rho}{8} A_{1}+\left(\frac{m+1}{3}\right) \rho^{3} A_{2}\right.$

$\left.+\left(\frac{m+1}{3}\right)\left(\frac{m+3}{5}\right) \rho^{5} A_{3}\right]$

$\sum_{k=0}^{\infty} k^{3} \rho^{k} b_{k, m}=2 \Gamma\left(\frac{m+1}{2}\right)\left\{\rho A_{1}+26\left(\frac{m+1}{3}\right) \rho^{3} A_{2}\right.$

(x) $\quad+72\left(\frac{m+1}{3}\right)\left(\frac{m+3}{5}\right) \rho^{5} A_{3}$

$\left.+48\left(\frac{m+1}{3}\right)\left(\frac{m+3}{5}\right)\left(\frac{m+5}{7}\right) \rho^{7} A_{4}\right\}$

$\sum_{k=0}^{\infty} k^{4} \rho^{k} b_{k, m}=4 \Gamma\left(\frac{m+1}{2}\right)\left\{\frac{\rho}{2} A_{1}+40\left(\frac{m+1}{3}\right) \rho^{3} A_{2}\right.$

$+262\left(\frac{m+1}{3}\right)\left(\frac{m+3}{5}\right) \rho^{5} A_{3}$

(xi)

$$
\left.\begin{array}{l}
+380\left(\frac{m+1}{3}\right)\left(\frac{m+3}{5}\right)\left(\frac{m+5}{7}\right) \rho^{7} A_{4} \\
+192\left(\frac{m+1}{3}\right)\left(\frac{m+3}{5}\right)\left(\frac{m+5}{7}\right)\left(\frac{m+7}{9}\right) \rho^{9} A_{5}
\end{array}\right\}
$$

(xii)

$$
\begin{aligned}
& \sum_{k=0}^{\infty} k^{\{2\}} \rho^{k} b_{k, m}=\Gamma\left(\frac{m+1}{2}\right)\left(\frac{m+1}{3}\right) \\
& \times\left[12 \rho^{3} A_{2}+\left(\frac{m+3}{5}\right) \rho^{5} A_{3}\right]
\end{aligned}
$$

(xiii)

$$
\sum_{k=0}^{\infty} k^{\{3\}} \rho^{k} b_{k, m}=4 \Gamma\left(\frac{m+1}{2}\right)\left(\frac{m+1}{3}\right)
$$

$\times\left[\rho^{3} A_{2}+6\left(\frac{m+3}{5}\right) \rho^{5} A_{3}+12\left(\frac{m+3}{5}\right)\left(\frac{m+5}{7}\right) \rho^{7} A_{4}\right]$

$\sum_{k=0}^{\infty} k^{\{4\}} \rho^{k} b_{k, m}=8 \Gamma\left(\frac{m+1}{2}\right)\left(\frac{m+1}{3}\right)\left(\frac{m+3}{5}\right)$
$\times\left\{45 \rho^{5} A_{3}+118\left(\frac{m+5}{7}\right) \rho^{7} A_{4}+96\left(\frac{m+5}{7}\right)\left(\frac{m+7}{9}\right) \rho^{9} A_{5}\right\}$

Where:

$$
L(m, \rho)=\sqrt{\pi} \Gamma\left(\frac{m}{2}\right)\left(1-\rho^{2}\right)^{-\frac{m}{2}}
$$

$$
k^{\{c\}}=k(k-1) \ldots(k-c+1), k^{\{0\}}=1
$$

$$
\begin{aligned}
& w_{(3)}(m, \rho)=\left[\left(m^{3}+3 m^{2}+2 m\right) \rho^{6}+\left(3 m^{2}+6 m\right) \rho^{4}\right] \\
& \times\left(1-\rho^{2}\right)^{-3}
\end{aligned}
$$




$$
\begin{gathered}
w_{\{4\}}(m, \rho)=m\left[\left(m^{3}+6 m^{2}+11 m+6\right) \rho^{8}\right. \\
\left.+\left(6 m^{2}+30 m+36\right) \rho^{6}+(3 m+6) \rho^{4}\right]\left(1-\rho^{2}\right)^{-4} \\
w_{3}(m, \rho)=\left[4 m \rho^{2}+\left(6 m^{2}+4 m\right) \rho^{4}+m^{3} \rho^{6}\right]\left(1-\rho^{2}\right)^{-3} \\
w_{4}(m, \rho)=\left[\left(m^{4}+18 m^{2}+12 m\right) \rho^{8}\right. \\
+\left(12 m^{3}-20 m^{2}+8 m\right) \rho^{6} \\
\left.+\left(46 m^{2}-4 m\right) \rho^{4}+32 m \rho^{2}\right]\left(1-\rho^{2}\right)^{-4} \\
A_{1}={ }_{2} F_{1}\left(1,(m+1) / 2 ; 3 / 2 ; \rho^{2}\right) ; \\
A_{2}={ }_{2} F_{1}\left(2, \frac{m+3}{2} ; \frac{5}{2} ; \rho^{2}\right) ; \\
A_{3}={ }_{2} F_{1}\left(3, \frac{m+5}{2} ; \frac{7}{2} ; \rho^{2}\right) \\
A_{4}={ }_{2} F_{1}\left(4, \frac{m+7}{2} ; \frac{9}{2} ; \rho^{2}\right) ; \\
A_{5}={ }_{2} F_{1}\left(5, \frac{m+9}{2} ; \frac{11}{2} ; \rho^{2}\right)
\end{gathered}
$$

\section{Proof}

When $\mathrm{c}$ is even, we have $b_{k, m}=\frac{(2)^{k}}{k !} \Gamma\left(\frac{k+1}{2}\right) \Gamma\left(\frac{k+m}{2}\right), m>2, k>0$ and under this scenario the proof was provided by Joarder (2006).

If $\mathrm{c}$ is odd, let $z=\rho^{2}$, then:

$$
\sum_{k=0}^{\infty} z^{k / 2} b_{k, m}=2 z^{1 / 2} \Gamma\left(\frac{m+1}{2}\right)_{2} F_{1}\left(1, \frac{m+1}{2} ; \frac{3}{2} ; z\right)
$$

Differencing identity (9) with respect to $z$, we got (viii):

$$
\begin{aligned}
& \sum_{k=0}^{\infty}\left(\frac{k}{2}\right) z^{\left(\frac{k}{2}-1\right)} b_{k, m}=2 \Gamma\left(\frac{m+1}{2}\right)\left[\frac{1}{2} z^{-\frac{1}{2}}{ }_{2} F_{1}\left(1, \frac{m+1}{2} ; \frac{3}{2} ; z\right)\right. \\
& \left.+z^{\left(\frac{1}{2}\right)}\left(\frac{m+1}{3}\right){ }_{2} F_{1}\left(2, \frac{m+3}{2} ; \frac{5}{2} ; z\right)\right] \\
& \sum_{k=0}^{\infty} k z^{\left(\frac{k}{2}\right)} b_{k, m}=4 \Gamma\left(\frac{m+1}{2}\right)\left[\frac{1}{2} z^{\frac{1}{2}}{ }_{2} F_{1}\left(1, \frac{m+1}{2} ; \frac{3}{2} ; z\right)\right. \\
& \left.+z^{\left(\frac{3}{2}\right)}\left(\frac{m+1}{3}\right){ }_{2} F_{1}\left(2, \frac{m+3}{2} ; \frac{5}{2} ; z\right)\right]
\end{aligned}
$$
(ix):

Differencing now (10) with respect to $z$, we obtain

$$
\begin{aligned}
& \sum_{k=0}^{\infty} k\left(\frac{k}{2}\right) z^{\left(\frac{k}{2}-1\right)} b_{k, m}=4 \Gamma\left(\frac{m+1}{2}\right) \\
& \times\left[\frac{1}{4} z^{-\frac{1}{2}}{ }_{2} F_{1}\left(1, \frac{m+1}{2} ; \frac{3}{2} ; z\right)\right. \\
& +\frac{1}{2} z^{\frac{1}{2}}\left(\frac{m+1}{3}\right){ }_{2} F_{1}\left(2, \frac{m+3}{2} ; \frac{5}{2} ; z\right) \\
& +\left(\frac{m+1}{3}\right) \frac{3}{2} z^{\left(\frac{1}{2}\right)}{ }_{2} F_{1}\left(2, \frac{m+3}{2} ; \frac{5}{2} ; z\right) \\
& \left.+\left(\frac{m+1}{3}\right) z^{\left(\frac{3}{2}\right)} 2\left(\frac{m+3}{5}\right){ }_{2} F_{1}\left(3, \frac{m+5}{2} ; \frac{7}{2} ; z\right)\right]
\end{aligned}
$$$$
\sum_{k=0}^{\infty} k^{2} z^{\left(\frac{k}{2}\right)} b_{k, m}=\Gamma\left(\frac{m+1}{2}\right)\left[2 z^{\frac{1}{2}}{ }_{2} F_{1}\left(1, \frac{m+1}{2} ; \frac{3}{2} ; z\right)\right.
$$$$
+16\left(\frac{m+1}{3}\right) z^{\frac{3}{2}} F_{1}\left(2, \frac{m+3}{2} ; \frac{5}{2} ; z\right)
$$

$$
\left.+16\left(\frac{m+1}{3}\right)\left(\frac{m+3}{5}\right) z^{\left(\frac{5}{2}\right)}{ }_{2} F_{1}\left(3, \frac{m+5}{2} ; \frac{7}{2} ; z\right)\right]
$$

Differencing (11) with respect to $z$, we obtain (x):

$$
\begin{aligned}
& \sum_{k=0}^{\infty} k^{2}\left(\frac{k}{2}\right) z^{\left(\frac{k}{2}-1\right)} b_{k, m}=\Gamma\left(\frac{m+1}{2}\right)\left\{z^{-\frac{1}{2}}{ }_{2} F_{1}\left(1, \frac{m+1}{2} ; \frac{3}{2} ; z\right)\right. \\
& +2 z^{\frac{1}{2}}\left(\frac{m+1}{3}\right){ }_{2} F_{1}\left(2, \frac{m+3}{2} ; \frac{5}{2} ; z\right) \\
& +24\left(\frac{m+1}{3}\right) z^{\frac{1}{2}} F_{2}\left(2, \frac{m+3}{2} ; \frac{5}{2} ; z\right) \\
& +32\left(\frac{m+1}{3}\right) z^{\frac{3}{2}}\left(\frac{m+3}{5}\right){ }_{2} F_{1}\left(3, \frac{m+5}{2} ; \frac{7}{2} ; z\right) \\
& +16\left(\frac{m+1}{3}\right)\left(\frac{m+3}{5}\right)\left[\frac{5}{2} z^{\left(\frac{3}{2}\right)} F_{1}\left(3, \frac{m+5}{2} ; \frac{7}{2} ; z\right)\right. \\
& \left.\left.+3\left(\frac{m+5}{7}\right) z^{\left(\frac{5}{2}\right)}{ }_{2} F_{1}\left(4, \frac{m+7}{2} ; \frac{9}{2} ; z\right)\right]\right\}
\end{aligned}
$$

$$
\begin{aligned}
& \sum_{k=0}^{\infty} k^{3} z^{\left(\frac{k}{2}\right)} b_{k, m}=2 \Gamma\left(\frac{m+1}{2}\right)\left[z^{\frac{1}{2}} F_{1}\left(1, \frac{m+1}{2} ; \frac{3}{2} ; z\right)\right. \\
& +26\left(\frac{m+1}{3}\right) z^{\frac{3}{2}} F_{2}\left(2, \frac{m+3}{2} ; \frac{5}{2} ; z\right) \\
& +72\left(\frac{m+1}{3}\right)\left(\frac{m+3}{5}\right) z^{\frac{5}{2}} F_{1}\left(3, \frac{m+5}{2} ; \frac{7}{2} ; z\right) \\
& \left.+48\left(\frac{m+1}{3}\right)\left(\frac{m+3}{5}\right)\left(\frac{m+5}{7}\right) z^{\left(\frac{7}{2}\right)}{ }_{2} F_{1}\left(4, \frac{m+7}{2} ; \frac{9}{2} ; z\right)\right]
\end{aligned}
$$
(xi):

Differencing now (12) with respect to $z$, we obtain 


$$
\begin{aligned}
& \sum_{k=0}^{\infty} k^{3}\left(\frac{k}{2}\right) z^{\left(\frac{k}{2}-1\right)} b_{k, m}=2 \Gamma\left(\frac{m+1}{2}\right)\left\{\frac{1}{2} z^{-\frac{1}{2}}{ }_{2} F_{1}\left(1, \frac{m+1}{2} ; \frac{3}{2} ; z\right)\right. \\
& +z^{\frac{1}{2}}\left(\frac{m+1}{3}\right){ }_{2} F_{1}\left(2, \frac{m+3}{2} ; \frac{5}{2} ; z\right) \\
& +26\left(\frac{m+1}{3}\right)\left[\frac{3}{2} z^{\left(\frac{1}{2}\right)}{ }_{2} F_{1}\left(2, \frac{m+3}{2} ; \frac{5}{2} ; z\right)\right. \\
& \left.+z^{\frac{3}{2}} 2\left(\frac{m+3}{5}\right){ }_{2} F_{1}\left(3, \frac{m+5}{2} ; \frac{7}{2} ; z\right)\right] \\
& +72\left(\frac{m+1}{3}\right)\left(\frac{m+3}{5}\right)\left[\frac{5}{2} z^{\left(\frac{3}{2}\right)}{ }_{2} F_{1}\left(3, \frac{m+5}{2} ; \frac{7}{2} ; z\right)\right. \\
& \left.+z^{\frac{5}{2}} 3\left(\frac{m+5}{7}\right){ }_{2} F_{1}\left(4, \frac{m+7}{2} ; \frac{9}{2} ; z\right)\right] \\
& +48\left(\frac{m+1}{3}\right)\left(\frac{m+3}{5}\right)\left(\frac{m+5}{7}\right)\left[\frac{7}{2} z^{\left(\frac{5}{2}\right)}{ }_{2} F_{1}\left(4, \frac{m+7}{2} ; \frac{9}{2} ; z\right)\right. \\
& \left.\left.+380\left(\frac{m+1}{3}\right)\left(\frac{m+3}{3}\right)\left(\frac{m+5}{7}\right)\left(\frac{m+7}{9}\right){ }_{2} F_{1}\left(5, \frac{m+9}{2} ; \frac{11}{2} ; z\right)\right]\right\} \\
& +40\left(\frac{m+1}{3}\right) z_{2}^{\frac{3}{2}}{ }_{2} F_{1}\left(2, \frac{m+3}{2} ; \frac{5}{2} ; z\right) \\
& \sum_{k=0}^{\infty} k^{4} z^{\left(\frac{k}{2}\right)} b_{k, m}=4 \Gamma\left(\frac{m+3}{5}\right) z_{2}^{\frac{5}{2}} F_{2}\left(3, \frac{m+5}{2} ; \frac{m+7}{2} ; z\right)\left[\frac{9}{2} ; z\right) \\
& +\frac{1}{2} z_{2}^{\frac{1}{2}} F_{1}\left(1, \frac{m+1}{2} ; \frac{3}{2} ; z\right)
\end{aligned}
$$

Finally, (xii), (xiii) and (xiv) can be obtained by notice the following:

$$
\begin{aligned}
& \sum_{k=0}^{\infty} k^{\{2\}} \rho^{k} b_{k, m}=\sum_{k=0}^{\infty} k^{2} \rho^{k} b_{k, m}-\sum_{k=0}^{\infty} k \rho^{k} b_{k, m} \\
& \sum_{k=0}^{\infty} k^{\{3\}} \rho^{k} b_{k, m}=\sum_{k=0}^{\infty}\left(k^{3}-k^{2}+2 k\right) \rho^{k} b_{k, m} \\
& \sum_{k=0}^{\infty} k^{\{4\}} \rho^{k} b_{k, m}=\sum_{k=0}^{\infty}\left(k^{4}-6 k^{3}+11 k^{2}-6 k\right) \rho^{k} b_{k, m}
\end{aligned}
$$

Next Theorem 4, will be useful to evaluate some product moments.

\section{Theorem 4}

For a finite $a, b$ :

$$
{ }_{2} F_{1}(a, b ; b-1 ; z)=(1-z)^{-a}+\frac{a z}{(b-1)}(1-z)^{-(a+1)}
$$

\section{Proof}

Note that:

$$
{ }_{2} F_{1}(a, b ; b-1 ; z)=\sum_{k=0}^{\infty} \frac{(a)_{k} \frac{(b+k-1) \Gamma(b+k-1)}{\Gamma(b)} z^{k}}{\frac{\Gamma(b-1+k)}{\Gamma(b-1)} k !}
$$

If we let $j=k$ - 1 , the above hypergeometric function can expressed as:

$$
\begin{aligned}
& { }_{2} F_{1}(a, b ; b-1 ; z) \\
& =\frac{\Gamma(b-1)}{\Gamma(b)}\left[\begin{array}{l}
\sum_{k=0}^{\infty} \frac{(b-1)(a)_{k} z^{k}}{k !}+\lim _{x \rightarrow 0} \frac{1}{\Gamma(x)} \\
+z \sum_{j=0}^{\infty} \frac{\Gamma(a+1+j)}{\frac{\Gamma(a+1)}{(a)}} \frac{z^{j}}{(j) !}
\end{array}\right]
\end{aligned}
$$

Now consider that (Ngo, 2012):

$$
\lim _{x \rightarrow 0}\left(\frac{1}{x}-\Gamma(x)\right)=\lim _{x \rightarrow 0}\left(-\frac{1}{x}-\Gamma(-x)\right)=\gamma
$$

where, $\gamma$ is the Euler's constant:

$$
\gamma=-\int_{0}^{\infty} e^{-x} \ln (x) d x \approx 0.57721566
$$

So the gamma function behaves almost exactly like $\left(\frac{1}{x}\right)$ when $x$ gets close to zero and $\frac{1}{\Gamma(x)}$ behaves almost exactly like zero when $x$ gets close to zero, so we have:

$$
{ }_{2} F_{1}(a, b ; b-1 ; z)={ }_{1} F_{0}(a ; ; z)+\frac{a z}{(b-1)} F_{1}(a+1 ; ; z)
$$

Some examples of product moments are given below For $a=\frac{1}{2}, b=\frac{1}{2}$ and $c=1$ we have:

$$
\begin{aligned}
& E\left[S_{1} S_{2} R\right]=\frac{\left(1-\rho^{2}\right)^{1+\frac{m}{2}}}{\sqrt{\pi} \Gamma\left(\frac{m}{2}\right) m} \sigma_{1} \sigma_{2} \rho \Gamma\left(\frac{m+2}{2}\right) \Gamma\left(\frac{3}{2}\right) \\
& \times{ }_{3} F_{2}\left(\frac{m+2}{2}, \frac{m+2}{2}, \frac{3}{2} ; \frac{m+2}{2}, \frac{3}{2} ; \rho^{2}\right) \\
& =\frac{1}{4} \sigma_{1} \sigma_{2} \rho
\end{aligned}
$$

For $a=\frac{1}{2}, b=\frac{3}{2}$ and $c=1$ we have: 


$$
\begin{aligned}
& E\left[S_{1} S_{2}^{3} R\right]=\frac{2\left(1-\rho^{2}\right)^{2+\frac{m}{2}}}{\sqrt{\pi} \Gamma\left(\frac{m}{2}\right) m^{2}} \sigma_{1} \sigma_{2}^{3} \rho \Gamma\left(\frac{m+4}{2}\right) \Gamma\left(\frac{3}{2}\right) \\
& \times_{3} F_{2}\left(\frac{m+2}{2}, \frac{m+4}{2}, \frac{3}{2} ; \frac{m+2}{2}, \frac{3}{2} ; \rho^{2}\right) \\
& =\left(1-\rho^{2}\right)^{2+\frac{m}{2}} \sigma_{1} \sigma_{2}^{3} \rho \frac{m(m+1){ }_{1} F_{0}\left(\frac{m}{2}+2 ; ; \rho^{2}\right)}{4 m^{2}} \\
& =\frac{(m+1)}{4 m} \sigma_{1} \sigma_{2}^{3} \rho
\end{aligned}
$$

For $a=1, b=1$ and $c=3$ we have:

$$
\begin{aligned}
& E\left[S_{1}^{2} S_{2}^{2} R^{2}\right]=\frac{4\left(1-\rho^{2}\right)^{\frac{4+m}{2}}}{\sqrt{\pi} \Gamma\left(\frac{m}{2}\right) m^{2}} \sigma_{1}^{2} \sigma_{2}^{2} \Gamma\left(\frac{m+2}{2}\right) \Gamma\left(\frac{m+2}{2}\right) \Gamma\left(\frac{3}{2}\right) \\
& \times \Gamma^{-1}\left(\frac{m+2}{2}\right){ }_{3} F_{2}\left(\frac{m+2}{2}, \frac{m+2}{2}, \frac{3}{2} ; \frac{m+2}{2}, \frac{1}{2} ; \rho^{2}\right) \\
& =\frac{\left(1-\rho^{2}\right)^{\frac{4+m}{2}}}{m} \sigma_{1}^{2} \sigma_{22}^{2} F_{1}\left(\frac{m+2}{2}, \frac{3}{2} ; \frac{1}{2} ; \rho^{2}\right) \\
& =\frac{\left(1-\rho^{2}\right)^{\frac{4+m}{2}}}{m} \sigma_{1}^{2} \sigma_{2}^{2} \\
& \times\left\{\left(1-\rho^{2}\right)^{-\frac{m+2}{2}}+(m+2) \rho^{2}\left(1-\rho^{2}\right)^{-\frac{m+4}{2}}\right\} \\
& =\frac{\sigma_{1}^{2} \sigma_{2}^{2}}{m}\left\{1+(m+1) \rho^{2}\right\}
\end{aligned}
$$

For $a=\frac{1}{2}, b=\frac{3}{2}$ and $c=3$ we have:

$$
\begin{aligned}
& E\left[S_{1} S_{2}^{3} R^{3}\right]=\frac{2\left(1-\rho^{2}\right)^{2+\frac{m}{2}}}{\sqrt{\pi} \Gamma\left(\frac{m}{2}\right) m^{2}} \sigma_{1} \sigma_{2}^{3} \rho \Gamma\left(\frac{m+2}{2}\right) \Gamma\left(\frac{5}{2}\right) \\
& \times{ }_{3} F_{2}\left(\frac{m+2}{2}, \frac{m+4}{2}, \frac{5}{2} ; \frac{m+4}{2}, \frac{3}{2} ; \rho^{2}\right) \\
& =\frac{3\left(1-\rho^{2}\right)^{2+\frac{m}{2}}}{4 m} \sigma_{1} \sigma_{2}^{3} \rho_{2} F_{1}\left(\frac{m+2}{2}, \frac{5}{2} ; \frac{3}{2} ; \rho^{2}\right) \\
& =\frac{3\left(1-\rho^{2}\right)^{2+\frac{m}{2}}}{4 m} \sigma_{1} \sigma_{2}^{3} \rho \\
& \times\left\{\left(1-\rho^{2}\right)^{-\frac{m+2}{2}}+\frac{m+2}{3} \rho^{2}\left(1-\rho^{2}\right)^{-\frac{m+4}{2}}\right\} \\
& =\frac{\sigma_{1} \sigma_{2}^{3} \rho}{4 m}\left(3+\rho^{2}(m-1)\right)
\end{aligned}
$$

For $a=1, b=\frac{1}{2}$ and $c=4$ we have:

$$
\begin{aligned}
& E\left[S_{1}^{2} S_{2} R^{4}\right]=\frac{2^{\frac{3}{2}}\left(1-\rho^{2}\right)^{\frac{3+m}{2}}}{\sqrt{\pi} \Gamma\left(\frac{m}{2}\right) m^{\frac{3}{2}}} \sigma_{1}^{2} \sigma_{2} \Gamma\left(\frac{m+2}{2}\right) \Gamma\left(\frac{m+1}{2}\right) \\
& \times \Gamma\left(\frac{5}{2}\right) \Gamma^{-1}\left(\frac{m+4}{2}\right) \times_{3} F_{2}\left(\frac{m+2}{2}, \frac{m+1}{2}, \frac{5}{2} ; \frac{m+4}{2}, \frac{1}{2} ; \rho^{2}\right) \\
& =\frac{(3) \sqrt{2}\left(1-\rho^{2}\right)^{\frac{3+m}{2}}}{(n+2) \Gamma\left(\frac{m}{2}\right) m^{\frac{3}{2}}} \sigma_{1}^{2} \sigma_{2} \Gamma\left(\frac{m+1}{2}\right) \\
& \times{ }_{3} F_{2}\left(\frac{m+2}{2}, \frac{m+1}{2}, \frac{5}{2} ; \frac{m+4}{2}, \frac{1}{2} ; \rho^{2}\right)
\end{aligned}
$$

\section{Corollary 1}

The moments of the sample correlation, if $c$ is even, are given by:

$$
\begin{aligned}
& E\left[R^{c}\right]=\frac{\left(1-\rho^{2}\right)^{\frac{n-1}{2}}}{\sqrt{\pi}} \Gamma\left(\frac{n-1}{2}\right) \Gamma\left(\frac{c+1}{2}\right) \Gamma^{-1}\left(\frac{n-1+c}{2}\right) \\
& \times_{3} F_{2}\left(\frac{n-1}{2}, \frac{n-1}{2}, \frac{c+1}{2} ; \frac{n-1+c}{2}, \frac{1}{2} ; \rho^{2}\right)
\end{aligned}
$$

If $c$ is odd, are given by:

$$
\begin{aligned}
& E\left[R^{c}\right]=\frac{2 \rho\left(1-\rho^{2}\right)^{\frac{n-1}{2}}}{\sqrt{\pi} \Gamma\left(\frac{n-1}{2}\right)}\left[\Gamma\left(\frac{n}{2}\right)\right]^{2} \Gamma\left(\frac{c+2}{2}\right) \Gamma^{-1}\left(\frac{n+c}{2}\right) \\
& \times{ }_{3} F_{2}\left(\frac{n}{2}, \frac{n}{2}, \frac{c+2}{2} ; \frac{n+c}{2}, \frac{3}{2} ; \rho^{2}\right)
\end{aligned}
$$

\section{Proof}

Use $a=0, b=0$ in Equation 3 and 4, then replace $m$ $=n-1$.

The first moment of $\mathrm{R}$, is obtained using, $c=1$ in Equation 16:

$$
\begin{aligned}
& E[R]=\frac{2 \rho\left(1-\rho^{2}\right)^{\frac{n-1}{2}}}{\sqrt{\pi} \Gamma\left(\frac{n-1}{2}\right)}\left[\Gamma\left(\frac{n}{2}\right)\right]^{2} \Gamma\left(\frac{3}{2}\right) \Gamma^{-1}\left(\frac{n+1}{2}\right) \\
& \times{ }_{3} F_{2}\left(\frac{n}{2}, \frac{n}{2}, \frac{3}{2} ; \frac{n+1}{2}, \frac{3}{2} ; \rho^{2}\right) \\
& =\frac{2 \rho\left(1-\rho^{2}\right)^{\frac{n-1}{2}}}{n-1}\left[\Gamma\left(\frac{n}{2}\right) \Gamma^{-1}\left(\frac{n-1}{2}\right)\right]_{3}^{2} F_{2}\left(\frac{n}{2}, \frac{n}{2} ; \frac{n+1}{2} ; \rho^{2}\right) \\
& =\frac{2 \rho}{n-1}\left[\Gamma\left(\frac{n}{2}\right) \Gamma^{-1}\left(\frac{n-1}{2}\right)\right]_{3}^{2} F_{2}\left(\frac{1}{2}, \frac{1}{2} ; \frac{n+1}{2} ; \rho^{2}\right) .
\end{aligned}
$$

Equation 17 is a well-known equation for the first moment of $R$ (Ghosh, 1966). 
The second, third and four moments of $R$, are obtained using, $c=2,3$ and 4 , depending the case:

$$
\begin{aligned}
& E\left[R^{2}\right]=\frac{\left(1-\rho^{2}\right)^{\frac{n-1}{2}}}{\sqrt{\pi}} \Gamma\left(\frac{n-1}{2}\right) \Gamma\left(\frac{3}{2}\right) \Gamma^{-1}\left(\frac{n+1}{2}\right) \\
& \times_{3} F_{2}\left(\frac{n-1}{2}, \frac{n-1}{2}, \frac{3}{2} ; \frac{n+1}{2}, \frac{1}{2} ; \rho^{2}\right) \\
& =\frac{\left(1-\rho^{2}\right)^{\frac{n-1}{2}}}{(n-1)}{ }_{3} F_{2}\left(\frac{n-1}{2}, \frac{n-1}{2}, \frac{3}{2} ; \frac{n+1}{2}, \frac{1}{2} ; \rho^{2}\right) \\
& E\left[R^{3}\right]=\frac{2 \rho\left(1-\rho^{2}\right)^{\frac{n-1}{2}}}{\sqrt{\pi} \Gamma\left(\frac{n-1}{2}\right)}\left[\Gamma\left(\frac{n}{2}\right)\right]^{2} \Gamma\left(\frac{5}{2}\right) \Gamma^{-1}\left(\frac{n+3}{2}\right) \\
& \times_{3} F_{2}\left(\frac{n}{2}, \frac{n}{2}, \frac{5}{2} ; \frac{n+3}{2}, \frac{3}{2} ; \rho^{2}\right) \\
& =\frac{3 \rho(n+1)\left(1-\rho^{2}\right)^{\frac{n-1}{2}}}{4} \frac{\Gamma\left(\frac{n}{2}\right)}{\Gamma\left(\frac{n-1}{2}\right)_{3}} F_{2}\left(\frac{n}{2}, \frac{n}{2}, \frac{5}{2} ; \frac{n+3}{2}, \frac{3}{2} ; \rho^{2}\right) \\
& E\left[R^{4}\right]=\frac{\left(1-\rho^{2}\right)^{\frac{n-1}{2}}}{\sqrt{\pi}} \Gamma\left(\frac{n-1}{2}\right) \Gamma\left(\frac{5}{2}\right) \Gamma^{-1}\left(\frac{n+3}{2}\right) \\
& \times_{3} F_{2}\left(\frac{n-1}{2}, \frac{n-1}{2}, \frac{5}{2} ; \frac{n+3}{2}, \frac{1}{2} ; \rho^{2}\right) \\
& =\frac{3\left(1-\rho^{2}\right)^{\frac{n-1}{2}}}{2(n-1)(n+1)}{ }_{3} F_{2}\left(\frac{n-1}{2}, \frac{n-1}{2}, \frac{5}{2} ; \frac{n+3}{2}, \frac{1}{2} ; \rho^{2}\right)
\end{aligned}
$$

In the literature there are expressions for the first four moments of R (Ghosh, 1966; Soper et al., 1917). The second, third and four moment of $\mathrm{R}$ may be expressed as:

$$
\begin{aligned}
& E\left[R^{2}\right]=1-\frac{(n-2)\left(1-\rho^{2}\right)}{n-1}{ }_{2} F_{1}\left(1,1 ; \frac{n+1}{2} ; \rho^{2}\right) \\
& E\left[R^{3}\right]=E[R]-\rho\left(1-\rho^{2}\right) \frac{2(n-2)}{(n+1)(n-1)} \\
& \times\left[\Gamma\left(\frac{n}{2}\right) \Gamma^{-1}\left(\frac{n-1}{2}\right)\right]_{2}^{2} F_{1}\left(\frac{3}{2}, \frac{3}{2} ; \frac{n+3}{2} ; \rho^{2}\right) \\
& E\left[R^{4}\right]=1+\frac{(n-2)(n-4)\left(1-\rho^{2}\right)}{2(n-1)}{ }_{2} F_{1}\left(1,1 ; \frac{n+1}{2} ; \rho^{2}\right) \\
& -\frac{n(n-2)\left(1-\rho^{2}\right)}{4 \rho^{2}}\left[{ }_{2} F_{1}\left(1,1 ; \frac{n+1}{2} ; \rho^{2}\right)-1\right]
\end{aligned}
$$

The equation of the third moment given by Ghosh (1966) is not correct. Equation 22 comes from the equation reported by Soper et al. (1917), which is

$$
\begin{aligned}
& E\left[R^{3}\right]=E[R]-\rho\left(1-\rho^{2}\right) \frac{q_{n+2}}{q_{n-1}} \frac{(n-2)}{n} \\
& \times\left[1+\frac{\rho^{2} 3^{2}}{2(n+3)}+\frac{\rho^{4} 3^{2} 5^{2}}{(4)(2)(n+3)(n+5)}\right. \\
& \left.+\frac{\rho^{6} 3^{2} 5^{2} 7^{2}}{(3 !)(8)(n+3)(n+5)(n+7)}+\ldots\right]
\end{aligned}
$$

Where:

$$
q_{n}=\int_{0}^{\pi / 2} \sin ^{n-1} \theta d \theta
$$

We note that:

$$
\begin{aligned}
& { }_{2} F_{1}\left(\frac{3}{2}, \frac{3}{2} ; \frac{n+3}{2} ; \rho^{2}\right)=1+\frac{\rho^{2} 3^{2}}{2(n+3)} \\
& +\frac{\rho^{4} 3^{2} 5^{2}}{(4)(2)(n+3)(n+5)} \\
& +\frac{\rho^{6} 3^{2} 5^{2} 7^{2}}{(3 !)(8)(n+3)(n+5)(n+7)}+\ldots
\end{aligned}
$$

And:

$$
\begin{aligned}
& \frac{q_{n+2}}{q_{n-1}}=\frac{\frac{\sqrt{\pi} \Gamma\left(\frac{n}{2}+1\right)}{2 \Gamma\left(\frac{n+1}{2}+1\right)}}{\frac{\sqrt{\pi} \Gamma\left(\frac{n-1}{2}\right)}{2 \Gamma\left(\frac{n}{2}\right)}}=\frac{\frac{n}{2}\left[\Gamma\left(\frac{n}{2}\right)\right]^{2}}{\left(\frac{n+1}{2}\right)\left(\frac{n-1}{2}\right)\left[\Gamma\left(\frac{n-1}{2}\right)\right]^{2}} \\
& =\frac{2 n\left[\Gamma\left(\frac{n}{2}\right) \Gamma^{-1}\left(\frac{n-1}{2}\right)\right]^{2}}{(n+1)(n-1)}
\end{aligned}
$$

The expressions for the second, third and four moment of $\mathrm{R}$, that we derived, are shorter than existing equations. Algebraically it is not easy to verify if Equation 18-20 are equal to Equation 21-23 respectively, as an exercise we wrote a program using the software $\mathrm{R}$ to get values of the above expectations for some parameters, the results obtained do not show differences between the equations derived here and the equations reported in the literature. In Table 1, we show some data reported by Soper et al. (1917) p. 337 and what we got with Equation 17-20. 
Table 1. Comparison of the first four moments of R, between data reported by Soper et al. (1917) and values obtained with Equation 17-20, when $\rho=0.6$ and $n=25$

\begin{tabular}{lll}
\hline & Values reported by Soper et al. (1917) & Values obtained by Equation $17-20$ \\
\hline$E\left[R^{1}\right]$ & 0.591825 & 0.59182508 \\
$E\left[R^{2}\right]$ & 0.368739 & 0.36873932 \\
$E\left[R^{3}\right]$ & 0.238293 & 0.23829348 \\
$E\left[R^{4}\right]$ & 0.158510 & 0.15851038 \\
\hline
\end{tabular}

Let $W=\frac{S_{1}^{2}}{S_{2}^{2}}$, be the ratio of two sample variances.

The statistic $\mathrm{W}$ is widely used to test the homogeneity of two variances and sometimes is useful to know the mean and variance of $\mathrm{W}$. With the next corollary 2 , we can obtain the mean and variance of $\mathrm{W}$.

\section{Corollary 2}

For $m>2 a$, the moments of the ratio of two sample variances, $W=\frac{S_{1}^{2}}{S_{2}^{2}}$, are given by:

$$
\begin{aligned}
& E\left[W^{a}\right]=\frac{\left(1-\rho^{2}\right)^{\frac{m}{2}}}{\left[\Gamma\left(\frac{m}{2}\right)\right]^{2}}\left(\frac{\sigma_{1}^{2}}{\sigma_{2}^{2}}\right)^{a} \Gamma\left(\frac{m+2 a}{2}\right) \Gamma\left(\frac{m-2 a}{2}\right) \\
& \times{ }_{2} F_{1}\left(\frac{m+2 a}{2}, \frac{m-2 a}{2} ; \frac{m}{2} ; \rho^{2}\right)
\end{aligned}
$$

\section{Proof}

Use $b=-a$ and $c=0$ in Equation 3.

The expectation of the ratio of two sample variances, $\mathrm{W}$, is given by:

$$
\begin{aligned}
& E[\mathrm{~W}]=\frac{\left(1-\rho^{2}\right)^{\frac{m}{2}}}{\left[\Gamma\left(\frac{m}{2}\right)\right]^{2}}\left(\frac{\sigma_{1}^{2}}{\sigma_{2}^{2}}\right) \Gamma\left(\frac{m+2}{2}\right) \Gamma\left(\frac{m-2}{2}\right) \\
& \times{ }_{2} F_{1}\left(\frac{m+2}{2}, \frac{m-2}{2} ; \frac{m}{2} ; \rho^{2}\right) \\
& =\frac{\left(\frac{m}{2}\right)\left(1-\rho^{2}\right)^{\frac{m}{2}}}{\left(\frac{m}{2}-1\right)}\left(\frac{\sigma_{1}^{2}}{\sigma_{2}^{2}}\right) \\
& \times\left[\left(1-\rho^{2}\right)^{-\frac{m+2}{2}}+\frac{(m+2) \rho^{2}}{(m-2)}\left(1-\rho^{2}\right)^{-\frac{m+4}{2}}\right] \\
& =\left(\frac{\sigma_{1}^{2}}{\sigma_{2}^{2}}\right) \frac{m}{(m-2)\left(1-\rho^{2}\right)}\left[1+\frac{(m+2) \rho^{2}}{(m-2)\left(1-\rho^{2}\right)}\right] \\
& =\left(\frac{\sigma_{1}^{2}}{\sigma_{2}^{2}}\right) \frac{m\left(m-2+4 \rho^{2}\right)}{\left[(m-2)\left(1-\rho^{2}\right)\right]^{2}}
\end{aligned}
$$

The second moment of the ratio $W$, is given by:

$$
\begin{aligned}
& E\left[W^{2}\right]=\frac{\left(1-\rho^{2}\right)^{\frac{m}{2}}}{\left[\Gamma\left(\frac{m}{2}\right)\right]^{2}}\left(\frac{\sigma_{1}^{2}}{\sigma_{2}^{2}}\right)^{2} \Gamma\left(\frac{m+4}{2}\right) \Gamma\left(\frac{m-4}{2}\right) \\
& \times_{2} F_{1}\left(\frac{m+4}{2}, \frac{m-4}{2} ; \frac{m}{2} ; \rho^{2}\right) \\
& =\frac{m(m+2)\left(1-\rho^{2}\right)^{\frac{m}{2}}}{(m-2)(m-4)}\left(\frac{\sigma_{1}^{2}}{\sigma_{2}^{2}}\right)^{2} F_{1}\left(\frac{m+4}{2}, \frac{m-4}{2} ; \frac{m}{2} ; \rho^{2}\right)
\end{aligned}
$$

The variance of $W$ is given by:

$$
\begin{aligned}
& \operatorname{Var}[W]=\frac{m(m+2)\left(1-\rho^{2}\right)^{\frac{m}{2}}}{(m-2)(m-4)}\left(\frac{\sigma_{1}^{2}}{\sigma_{2}^{2}}\right)_{2}^{2} F_{1}\left(\frac{m+4}{2}, \frac{m-4}{2} ; \frac{m}{2} ; \rho^{2}\right) \\
& -\left[\left(\frac{\sigma_{1}^{2}}{\sigma_{2}^{2}}\right) \frac{m\left(m-2+4 \rho^{2}\right)}{\left[(m-2)\left(1-\rho^{2}\right)\right]^{2}}\right]^{2} \\
& =\left(\frac{\sigma_{1}^{2}}{\sigma_{2}^{2}}\right)^{2} \frac{m}{(m-2)}\left[\frac{(m+2)\left(1-\rho^{2}\right)^{\frac{m}{2}}}{(m-4)}{ }_{2} F_{1}\left(\frac{m+4}{2}, \frac{m-4}{2} ; \frac{m}{2} ; \rho^{2}\right)\right. \\
& \left.-\frac{m\left(m-2+4 \rho^{2}\right)^{2}}{(m-2)^{3}\left(1-\rho^{2}\right)^{4}}\right]
\end{aligned}
$$

In the case of $\rho=0$, we have:

$$
\begin{gathered}
E\left[\frac{S_{1}^{2}}{S_{2}^{2}}\right]=\left(\frac{\sigma_{1}^{2}}{\sigma_{2}^{2}}\right) \frac{m}{(m-2)} \\
\operatorname{Var}[W]=\left(\frac{\sigma_{1}^{2}}{\sigma_{2}^{2}}\right)^{2} \frac{m}{(m-2)}\left[\frac{(m+2)}{(m-4)}-\frac{m}{(m-2)}\right] \\
=\left(\frac{\sigma_{1}^{2}}{\sigma_{2}^{2}}\right)^{2} \frac{2 m(2 m-2)}{(m-2)^{2}(m-4)}
\end{gathered}
$$

If furthermore, the two variances are equal the expectation and variance of $\mathrm{W}$ become the expectation and variance of a central F-distribution with parameters $d_{1}=d_{2}=m$. In the future, it will be useful to study the variance of $\mathrm{W}$ to test homogeneity of variances for two correlated samples. 


\section{Conclusion}

We derive a general result to get any product moments of the sample variances and sample correlation coefficient when the data come from a bivariate normal distribution, the final expression is given in terms of the hypergeometric function which is a well-known function and there exists computational routines to be evaluated. A general expression to get the moments of the correlation sample is obtained and a validation of the result was carried out. Finally and equation to get the moments of the ratio of two sample variances was derived and as a particular case, the expectation and variance of the ratio of two sample variances were obtained.

\section{Ethics}

This article is original and contains unpublished material. There are no ethical issues involved in any aspect of this paper.

\section{References}

Anderson, T.W., 2003. An Introduction to Multivariate Statistical Analysis. 1st Edn., Wiley-Interscience, ISBN-10: 0471360910, pp: 752.

Bailey, W.N., 1964. Generalized Hypergeometric Series. 1st Edn., Hafner, New York, pp: 108.
Ghosh, B.K., 1966. Asymptotic expansions for the moments of the distribution of correlation coefficient. Biometrika, 53: 258-262. DOI: $10.2307 / 2334076$

Joarder, A.H., 2006. Product moments of a bivariate Wishart distribution. J. Probability Stat. Sci., 4: 233-244.

Ngo, T.M., 2012. On the behavior of the gamma function on the negative side. Senior Honors Theses, University of New Orleans, Louisiana, USA.

Oberhettinger, F., 1970. Hypergeometric Functions. In: Handbook of Mathematical Functions with Formulas, Graphs and Mathematical Tables, Abramowitz, M. and I. Stegun (Eds.), Dover publications, Inc. New York, ISBN-10: 486612724, pp: 555-566.

Rady, E.A., H.A. Fergany and A.M. Edress, 2005. An easy way to calculate the moments of sample correlation coeficients. Commun. Fac. Sci. Univ. Ank. Series A1. 54: 21-28.

Soper, H.E., A.W. Young, B.M. Cave, A. Lee and K. Pearson, 1917. On the distribution of the correlation coefficient in small samples. Appendix II to the papers of "student" and R. A. fisher. Biometrika, 11: 328-413. DOI: $10.2307 / 2331830$ 